\title{
Hydrothermal liquefaction of wood chips under supercritical and subcritical water reaction conditions
}

\author{
Naoto Shimizu ${ }^{1,2} \cdot$ Bingyao Zeng $^{3} \cdot$ Keisuke Kushima ${ }^{3}$
}

Received: 28 December 2020 / Accepted: 7 April 2021

Published online: 23 April 2021

(c) The Author(s) 2021, corrected publication 2021 OPEN

\begin{abstract}
This work describes batch-type hydrothermal liquefaction (HTL) treatments of conifer wood chips at $180-425^{\circ} \mathrm{C}$, under either air or nitrogen atmosphere. Such experiments allow efficient extraction of 5-hydroxymethyl furfural (HMF) and other valuable chemical substances, such as glycolic acid and acetic acid, from the lignocellulosic biomass. These compounds and their decomposition products present in the samples after HTL are analyzed and quantified using spectroscopic and chromatographic techniques. In general, the relatively higher-pressure nitrogen atmospheric condition is more suitable for obtaining the desired products, relative to the air atmosphere. Based on the quantitative results, the optimal temperatures for producing acetic acid, glycolic acid, and $\mathrm{HMF}$ are $300^{\circ} \mathrm{C}, 250^{\circ} \mathrm{C}$, and $180^{\circ} \mathrm{C}$, respectively. The interesting relationship between HMF yield and temperature is also discussed; as the temperature increases, the yield of HMF first decreases and then increases. This phenomenon is explained by the exothermic nature of the HMF decomposition reaction, which is inhibited by excessively high temperature (in the range from 380 to $425^{\circ} \mathrm{C}$ ). At moderately high temperatures (optimized conditions; $300^{\circ} \mathrm{C}$ ), the generation rate of HMF exceeds its decomposition rate, resulting in a high yield of HMF. Based on the results of the experiments conducted in this study, the decomposition mechanism describing HTL treatment of wood chips can be elucidated. This study therefore provides guidance for future work involving HMF extraction from lignocellulosic biomass.
\end{abstract}

Keywords Hydrothermal treatment · Lignocellulosic biomass · Bioenergy production · Biofuel $\cdot$ Hydroxymethylfurfural

\section{Introduction}

Since the Industrial Revolution, society and technology have developed rapidly by employing fossil fuel resources, consequently exacerbating global warming. Carbon dioxide emitted from fossil fuel combustion is considered one of the main contributors to the greenhouse effect, which has several serious consequences. In particular, the melting of Arctic sea ice causes rising sea levels, reduction of land area, and frequent typhoons and heavy rain. Additionally, rising global temperatures lead to biological extinction and intense forest fires. Furthermore, general climate change influences agricultural production [1]. Recent efforts to reduce dependence on fossil fuel resources have inspired scientists to search for new available energy sources.

To address modern societal energy demands, numerous studies have investigated the use of clean, sustainable energy sources, such as solar, wind, and hydropower. However, the availability and intensity of solar and wind energy are governed by the weather, which leads to unstable out put. Similarly, hydroelectric power generation is impacted

\footnotetext{
$\triangle$ Naoto Shimizu, shimizu@bpe.agr.hokudai.ac.jp | ${ }^{1}$ Agriculture Engineering Laboratory, Research Faculty of Agriculture, Hokkaido University, Kita 9, Nishi 9, Kita-ku, Sapporo, Hokkaido 060-8589, Japan. ${ }^{2}$ Field Science Center for Northern Biosphere, Hokkaido Univ, Sapporo, Hokkaido 060-8589, Japan. ${ }^{3}$ Graduate School of Agriculture, Hokkaido Univ, Sapporo, Hokkaido 060-8589, Japan.
} 
by hydrology and geomorphology. In contrast, energy sources derived from biomass do not face any of these shortcomings [2]. Biomass is plant or animal material that can be used (1) to generate electrical or thermal energy, or (2) as the raw material in various industrial manufacturing processes [3]. The most notable advantages of using biomass for energy-related purposes are its abundance, availability, and carbon-neutral nature. This means that the carbon dioxide fixed by plants through photosynthesis is emitted during biofuel combustion, such that the total carbon dioxide emission is equal to zero; this aspect helps mitigate the greenhouse effect. The fuel produced using biomass is called biofuel, which can be classified as either first- or second-generation biofuel. First-generation biofuel is primarily extracted from corn, sugar cane, sugar beet, and other crops. These resources have a high planting density, resulting lower energy input. However, production of such resources for use as first-generation biofuels can incur high prices due to competition with food. Therefore, second-generation biofuels, which use inedible plants as raw materials, have attracted attention in recent years. One disadvantage associated with second-generation biofuel is the high energy input required to collect the material, which has low energy density. Consequently, an important aspect of employing second-generation biofuels involves finding methods to efficiently extract more useful substances from small amounts of raw materials.

Biomass is not only used for energy production, but also for manufacturing value-added products. In Japan, eight million tons of forest residue is generated every year, and only $9 \%$ of it is utilized further [4]. Wood chips are often used in experimental studies into the decomposition behavior of forest residue, as in the present work $[5,6]$. In Hokkaido, the tree species composition of the planted forest is $97 \%$ conifer and 3\% broadleaf in terms of area. Which shows that the utilization of conifers is more important [7]. A typical conifer wood chip contains $31 \sim 49 \%$ cellulose, $9 \sim 23 \%$ hemicellulose, and $20 \sim 35 \%$ lignin [8]. Various compounds, such as 5-hydroxymethyl furfural (HMF), acetic acid, glycolic acid, and others can be obtained following the decomposition of cellulose and hemicellulose. HMF is an important reagent for synthesizing dimethylformamide (DMF) [9], which can be used as a biofuel component, or to manufacture various chemicals, including caprolactam and 1,6-hexanediol, among others [10]. Acetic acid is another important chemical substance, which is used in the industrial production of calcium magnesium acetate $[11,12]$. Finally, glycolic acid can be used as a reagent for certain chemical analyses, or as a reagent in organic synthesis.

Hydrothermal treatments involve decomposing biomass in high-temperature and high-pressure water systems. This technology was used for the work presented herein to produce HMF and other valuable compounds because it can treat biomass without a drying process. Therefore, because of the high degree of moisture in biomass, this hydrothermal treatment strategy requires lower costs and is more energy efficient than other methods like pyrolysis and burning. Several reactions occur during hydrothermal treatment, including hydrolysis, dehydration, decarboxylation, polymerization, and condensation [13]. In hydrothermal treatment, reactions such as hydrolysis will be promoted, so the production of HMF, glycolic acid and acetic acid related to the hydrolysis reaction will be promoted. When the temperature and pressure of water are above its critical point $\left(374{ }^{\circ} \mathrm{C}\right.$ and $22.1 \mathrm{MPa}$, respectively), the phase boundary between gas and liquid disappears, and the water is considered to be in a supercritical state. Under these conditions, even minor temperature fluctuations can drastically alter the electrical conductivity and ionic product of the supercritical water, which ultimately influences the rate of reactions occurring in solution (i.e., hydrolysis). The distinct situation wherein a substance is below its critical point and above its boiling point is sometimes referred to as the "subcritical state" in the literature, although it is technically not a physical state. Because reaction rates in the supercritical versus subcritical states are significantly different, this study evaluates and compares experiments conducted under both types of conditions [14].

The temperature also has a significant influence on the efficacy of the hydrothermal treatment. Specifically, temperature directly impacts the rate of free radical reactions, and indirectly impacts the rate of ionic reactions by modifying the ionic product and dielectric constant of water [15-17]. The experiments in this study were conducted at different temperatures (from 180 to $425^{\circ} \mathrm{C}$ ) to determine the temperature dependence of the HMF, acetic acid, and glycolic acid yields. The high pressure required in such hydrothermal treatments is the main disadvantage because of the associated high cost of equipment [15]. To our knowledge, there are limited studies regarding the production of HMF via low-pressure hydrothermal treatment. Therefore, this report describes hydrothermal liquefaction (HTL) treatments of wood chips under either an air or nitrogen atmosphere, and evaluates the effect of pressure and temperature on the yield of each product (HMF, glycolic acid, acetic acid, and other substances) [18]. The decomposition of furfural and HMF during hydrothermal treatments has been widely studied [19-22], but the mutual conversion between organic acids, such as acetic acid and glycolic acid, is rarely mentioned. This study also clarifies the conversion pathways between organic acids and elucidates the decomposition mechanism of lignocellulosic biomass in hydrothermal reactions under air and nitrogen conditions. The objective of this research is to 
confirm the suitable condition for producing HMF, acetic acid and glycolic acid, and to investigate the influence of atmosphere and temperature on bio-oil production.

\section{Experimental section}

\subsection{Starting materials}

Conifer trees in northern Hokkaido (Japan) were used for the wood chip material in this study. The size of coniferous wood chip is about $0.2 \times 0.3 \times 1.6 \mathrm{~cm}^{3}$, and did not be sieved. HMF (98\%), glycolic acid (97\%), and acetic acid (99\%) standard samples and sulfuric acid were purchased from Wako Pure Chemical Industries, Ltd (Osaka, Japan). All of the standard samples were used as-received. Sulfuric acid was filtered with $0.45 \mu \mathrm{m}$ filter paper.

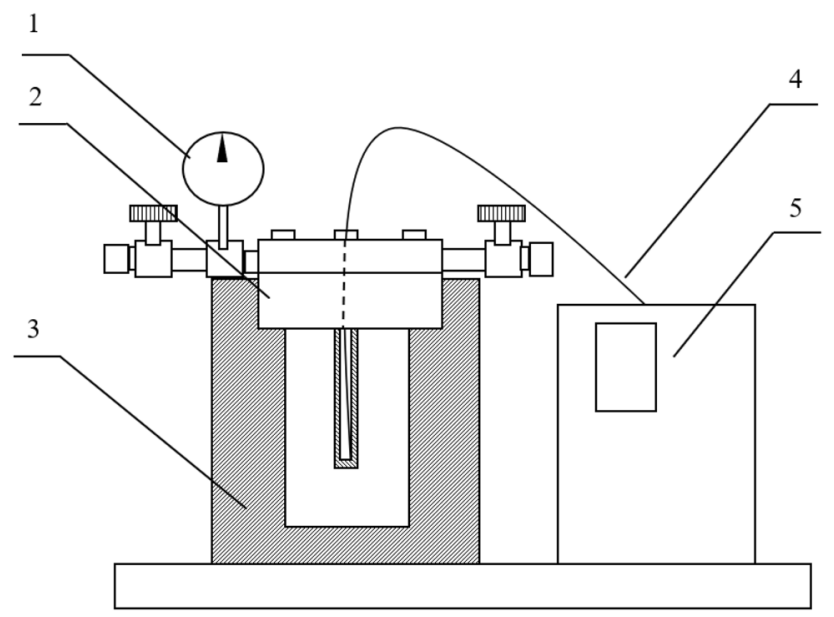

Fig. 1 Schematic diagram of a hydrothermal reactor. $1=$ pressure gauge, $2=$ reactor body, $3=$ heater, $4=$ thermocouple, $5=$ control unit

\subsection{Procedure}

Hydrothermal liquefaction treatments were performed in an autoclave (Model 122,841, SUS 316 Tsukuba, Japan) at various temperatures. Experiments conducted below $260^{\circ} \mathrm{C}$ used a reactor with a volume of $0.19 \mathrm{~L}$, and experiments conducted at temperatures above $260^{\circ} \mathrm{C}$ used a reactor with a volume of $0.16 \mathrm{~L}$. A diagram depicting the structure of the reactor is shown in Fig. 1. Batch tests were conducted at $180^{\circ} \mathrm{C}, 250^{\circ} \mathrm{C}, 300^{\circ} \mathrm{C}, 380^{\circ} \mathrm{C}$, and $425^{\circ} \mathrm{C}$, with initial pressures of $0.1 \mathrm{MPa}$ and $4 \mathrm{MPa}$. The experimental conditions are summarized in Table 1, and the experimental steps are outlined in Fig. 2. First, the biomass (wood chips) and water were loaded into the reactor at a designated biomass to water ratio (B/W ratio) of 1/10. For nitrogen atmosphere experiments, the autoclave was sealed and purged with $\mathrm{N}_{2}$ three times to remove residual air, then pressurized to $4 \mathrm{MPa}$ before beginning the heating process. In the air atmosphere experiments, this nitrogen purge was not performed. The reaction time is defined as the duration of the experiment after the autoclave

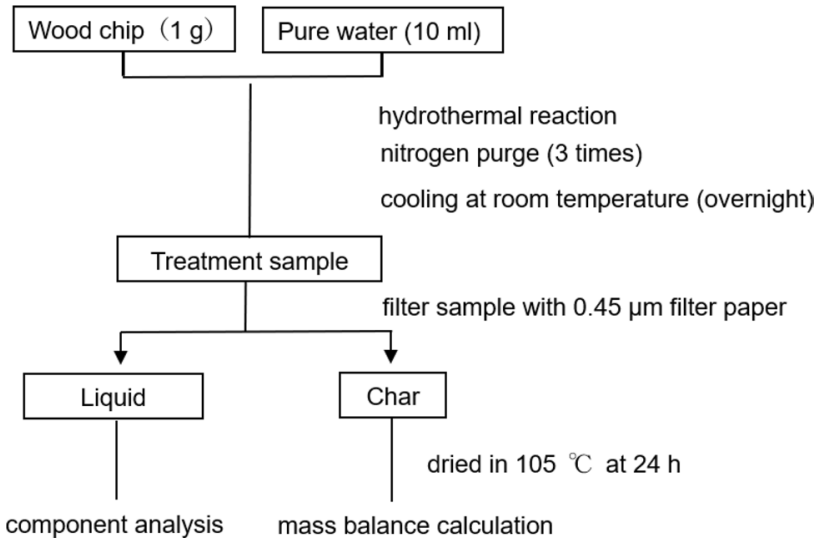

Fig. 2 Sample preparation for liquid component analysis and mass balance calculations
Table 1 Experimental conditions

\begin{tabular}{llllll}
\hline Material & $\begin{array}{l}\text { Temperature } \\
\left({ }^{\circ} \mathrm{C}\right)\end{array}$ & $\begin{array}{l}\text { Reaction time } \\
(\mathrm{min})\end{array}$ & Atmosphere & $\begin{array}{l}\text { Initial pressure } \\
(\mathrm{MPa})\end{array}$ & $\mathrm{B} / \mathrm{W}$ ratio \\
\hline Wood chip (1 g) & 180 & 30 & Nitrogen & 4.0 & $1 / 10$ \\
& 250 & 30 & & \\
& 300 & 5 & & \\
& 380 & 4 & Air & \\
425 & 0 & & \\
180 & 30 & & \\
250 & 30 & & \\
& 300 & 5 & & \\
& 380 & 4 & & \\
425 & 0 & &
\end{tabular}


temperature reached the target temperature. After stopping heating, the reactor is left overnight, and cooled to room temperature by air cooling. The samples were then collected and filtered with $0.45 \mu \mathrm{m}$ filter paper (mixed cellulose esters (MCE) membrane) to separate the liquid from the solid phase. Then, an electric furnace (FO300; Yamato scientific. Co, Ltd, Tokyo, Japan) was used to dry the char at $105^{\circ} \mathrm{C}$ for $24 \mathrm{~h}$ in air, and the mass balance was calculated. The liquid was analyzed quantitatively using high performance liquid chromatography (HPLC).

\subsection{Analysis}

\subsubsection{Mass balance}

The mass balance calculation is expressed by Eqs. 1, 2, and 3. After filtering and drying the char, the weight of the dry char is calculated as the total weight after drying minus the weight of the dry filter paper. Similarly, the total weight of the liquid (on the filter paper and char) can be calculated as the weights of char and filter before drying minus their weights after drying. The total liquid weight can be determined by adding the weight of the separated liquid portion. According to the principle of conservation of mass, the weight of the generated gas can be obtained by subtracting the weight of char and liquid from the sum of the total weight of pure water and biomass before the treatment.

$\mathrm{M}_{\text {solid }}=$ solid mass after drying

$M_{\text {liquid }}=$ measured liquid weight + solid mass before drying $-M_{\text {solid }}$

$M_{\text {gas }}=$ total material weight input $-M_{\text {solid }}-M_{\text {liquid }}$

\subsubsection{Carbon balance}

CHN analysis (CE440, Exeter Analytical, Inc, Coventry, UK) was conducted to confirm the carbon, nitrogen, oxygen and hydrogen content. The carbon content of raw materials, solid and liquid products is directly measured by $\mathrm{CHN}$ analysis. The carbon content of gas is calculated by Eq. 4. Each set of measurements is carried out twice, and the average value of the measurement results is used for graphing.

$\mathrm{C}_{\text {gas }}(\mathrm{g})=\mathrm{C}_{\text {wood chip }}(\mathrm{g})-\mathrm{C}_{\text {solid }}(\mathrm{g})-\mathrm{C}_{\text {liquid }}(\mathrm{g})$

\subsubsection{Absorbance spectra}

The optical absorbance spectra of the liquid samples were measured using a V-560 UV-visible spectrophotometer (JASCO V-560, JASCO corporation, Tokyo, Japan). All samples were diluted one hundred times with pure water before measurements. The changes in the color of the liquid can be used to estimate the extent of the reaction (Table 2).

\subsubsection{Qualitative and quantitative analysis of the liquid composition}

The composition of the liquid phase was analyzed using HPLC (1200 Infinity Series instrument equipped with a Shodex SUGAR SH 1821 column (Showa Denko, K.K.) and an Optilabrex 1260 GPC differential refractive index (RI) detector). Each sample was measured twice to obtain an average value for quantification. The standard deviation (SD) was also calculated. Sulfuric acid $\left(2.0 \times 10^{-3} \mathrm{~mol} / \mathrm{L}\right)$ was used as the mobile phase, with a flow rate of $0.6 \mathrm{~mL} / \mathrm{min}$, and the column temperature was maintained at $60^{\circ} \mathrm{C}$. The $\mathrm{RI}$ of each sample was also measured to obtain qualitative and quantitative analysis of each component.

Concentration (\%) of acetic acid, glycolic acid and HMF were quantified using absolute calibration curve method. The concentrations of acetic acid standard samples are $0.3 \%, 0.5 \%$ and $0.0 .7 \%$, glycolic acid standard samples are $0.3 \%, 0.5 \%$ and $0.0 .7 \%$, HMF standard samples are $0.02 \%$, $0.06 \%$ and $0.09 \%$. Use the linear regression method to find the linear regression equation, and calculate the concentration of each substance, the calculation formula is expressed by Eqs. 5, 6, and 7. Concentration ( $\mathrm{g} / \mathrm{L}$ ) and yield (g) are calculated with the Eq. 8.

acetic acid : concentration $(\%)=\frac{\text { area }+2.29}{70.25}$

glycolic acid : concentration $(\%)=\frac{\text { area }+10.82}{122.68}$

acetic acid : concentration $(\%)=\frac{\text { area }+4.3297}{281.05}$

concentration $(\mathrm{g} / \mathrm{L})=$ concentration $(\%) \times \operatorname{density}\left(\mathrm{g} / \mathrm{cm}^{3}\right) \times 10$ 
Table 2 Photographs of the liquid samples for each experiment

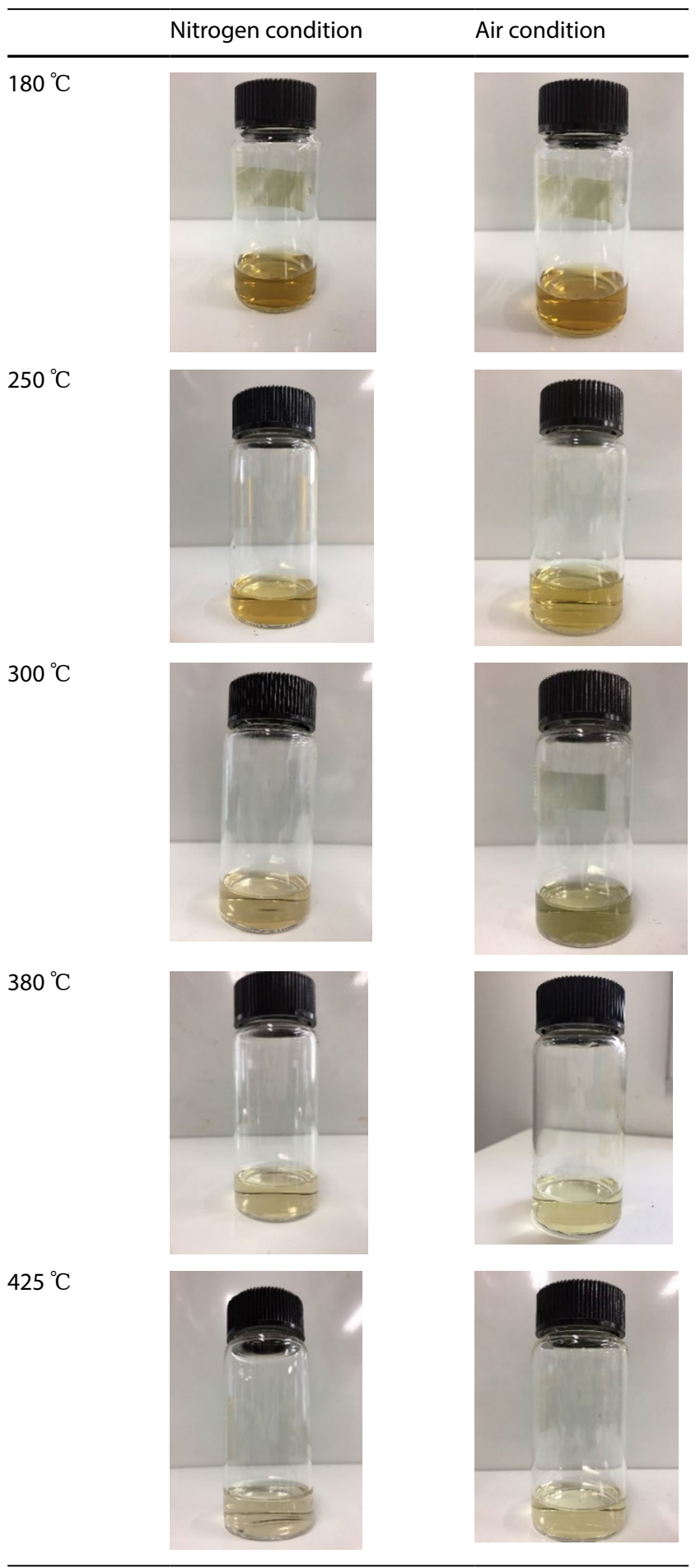

\section{Results and discussion}

\subsection{Supercritical/subcritical water reaction conditions}

In this work, the decomposition of lignocellulosic biomass was studied by conducting hydrothermal experiments on conifer wood chips (from Northern Hokkaido, Japan) under subcritical and supercritical water conditions. The temperature imposed during such treatments plays a vital role in the hydrothermal reactions. At low temperature, water molecules generate hydrogen bonds according to their polarity. Under these conditions, the intermolecular interactions are relatively strong, which makes it difficult for water molecules to participate in the reactions. However, as the temperature increases, the dielectric constant decreases [23-25], which indicates that the polarity of the water is also reduced. Therefore, water molecules dissociate to form hydronium ions $\left(\mathrm{H}_{3} \mathrm{O}^{+}\right)$, which cause the water to exhibit weakly acidic properties during the hydrothermal treatments. Additionally, formation of hydronium ions promotes the hydrolysis and depolymerization of hemicellulose $[13,26]$.

The temperature and pressure changes that occurred in each group of experiments are shown in Fig. 3. It is clear that the reaction pressure increased with rising temperature. As expected, higher reaction temperatures require more time for heating. In the hydrothermal experiments conducted at $380^{\circ} \mathrm{C}$ under an $\mathrm{N}_{2}$ atmosphere, the pressure did not reach $22 \mathrm{MPa}$ (the supercritical point), despite the fact that the reaction temperature exceeded the critical temperature. Therefore, a hydrothermal experimental temperature of $425{ }^{\circ} \mathrm{C}$ and a nitrogen atmosphere were required for the supercritical treatment $(22.5 \mathrm{MPa})$. The reaction time is defined as the duration of the experiment after the autoclave temperature reached the target temperature. After the reaction time exceeds the target reaction time, the heating is stopped, which obtained the decrease temperature.

\subsection{Mass balance}

The mass balance results from each experiment are provided in Fig. 4. Neither the process temperature nor the atmosphere had a significant influence on the mass 

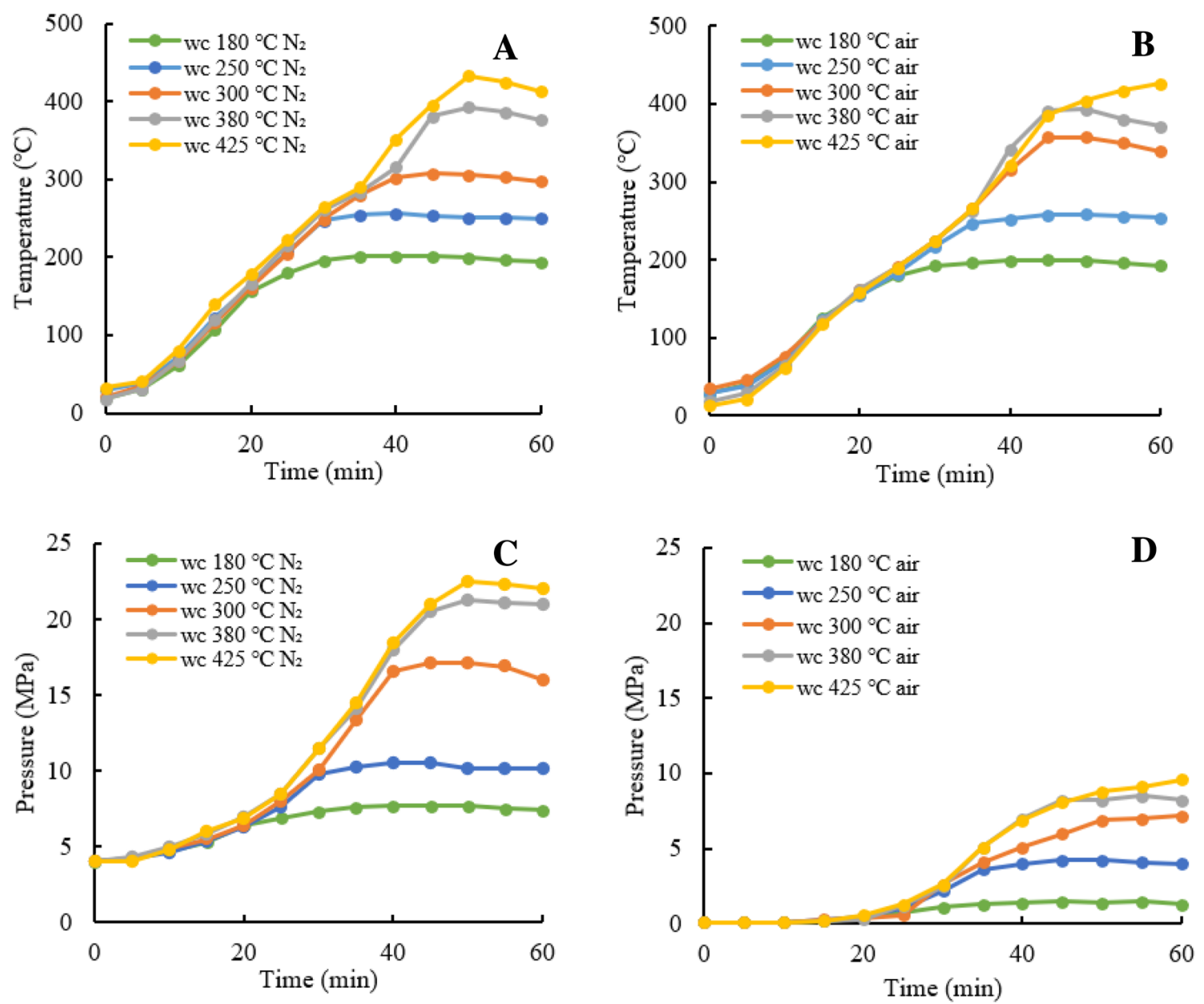

Fig. 3 Temperature-pressure changes in hydrothermal experiments under either $\mathrm{N}_{2}(\mathbf{a}, \mathbf{c})$ or air $(\mathbf{b}, \mathbf{d})$ atmospheres

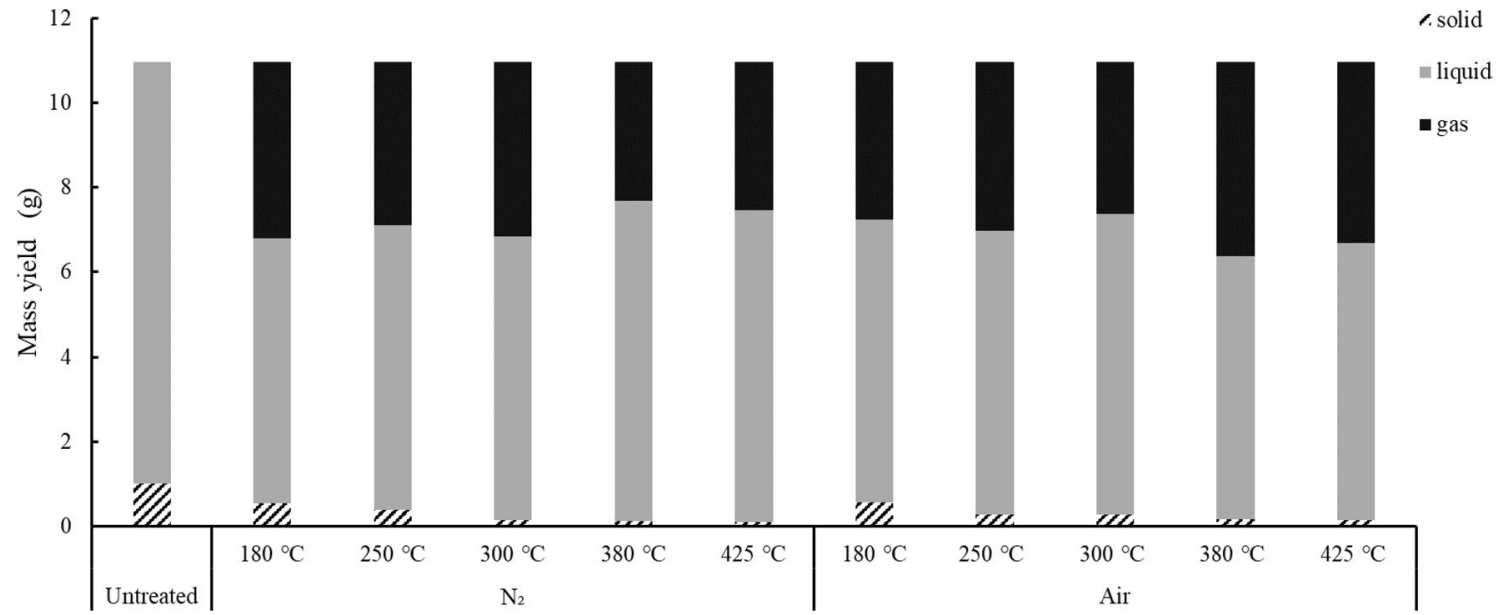

Fig. 4 Results of mass balance calculations for each group of experiments

balance. In addition, regardless of whether the experiment was conducted under air or nitrogen, the solid yield decreased with increasing process temperature. As mentioned previously, the formation of hydronium ions caused decomposition of hemicellulose and cellulose, leading to consumption of the solid phase. However, 
previous investigations determined that, under supercritical or subcritical conditions, either radical or ionic reactions are most prevalent, respectively [16]. Aromatic compounds are essential for producing char; however, as the temperature increases, the ionic reaction necessary for the formation of aromatic compounds is inhibited. Therefore, higher reaction temperatures hindered the generation of char [27], which reduced the overall solid yield. It is worth noting that, based on the mass balance calculated for hydrothermal experiments conducted with temperatures above $380^{\circ} \mathrm{C}$, the gas yield under air conditions is significantly higher than the gas yield under high-pressure nitrogen conditions. This may be because the oxygen in the air reacts with hydroxyl groups in HMF, glycolic acid, and other intermediates, causing them to decompose and produce more gas. The produced gas mixture is expected to include mainly hydrogen, carbon dioxide, methane, and carbon monoxide. However, the present study focuses on the HTL of wood chips, so we did not examine the gaseous components in detail. Other groups conducting similar research confirmed that the yield of hydrogen, carbon dioxide, carbon monoxide, and methane in the gas fraction are higher at supercritical conditions than at subcritical conditions [2].

\subsection{Carbon balance}

Elemental analysis was performed on the wood chip, the char and the liquid from $180{ }^{\circ} \mathrm{C}, 250{ }^{\circ} \mathrm{C}$ and $300{ }^{\circ} \mathrm{C}$ hydrothermal treatment under nitrogen conditions, and the results of the carbon balance are shown in Fig. 5. It can be confirmed that as the temperature rises, the mass of carbon in solid phase decreases. As the high temperature promotes the decomposition of organic compounds like cellulose and hemicellulose, more carbon is transferred from the wood to the gas phase. This may mean an increase in carbon monoxide or carbon dioxide emissions. Therefore,

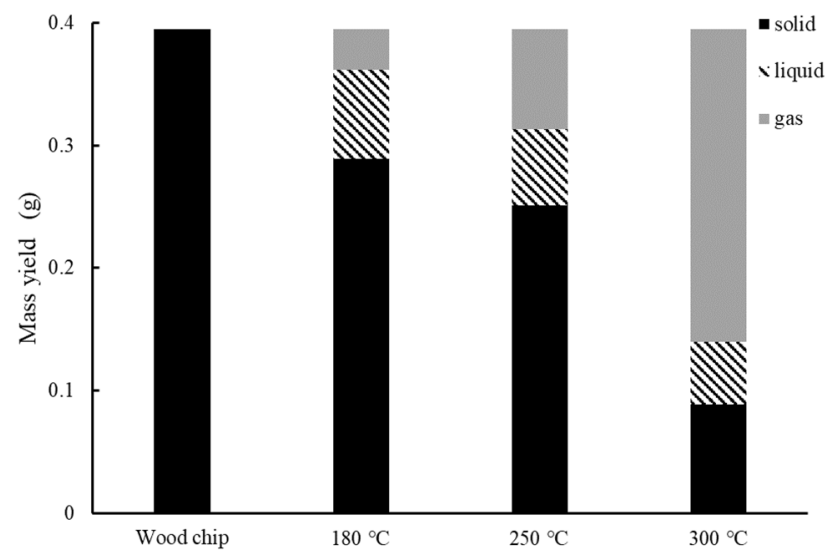

Fig. 5 Carbon balance for each group of experiments whether it is from the viewpoint of environmental or energy production, low temperature treatment should be given priority over high temperature treatment. On the other hand, as the temperature increases, the carbon content of the liquid has little effect.

\subsection{Absorption spectra}

The UV absorption profiles (in the range from 200 to $400 \mathrm{~nm}$ ) obtained for each liquid phase are given in Fig. 6 . The liquid sample obtained following hydrothermal treatment at $180^{\circ} \mathrm{C}$ displays the most intense absorbance at $\lambda_{\max }=282 \mathrm{~nm}$, and this peak was assigned to HMF [28]; the sample obtained following hydrothermal treatment at $425^{\circ} \mathrm{C}$ had the lowest absorbance at this wavelength. These results indicate that HMF components of these liquid samples are decomposed at high temperatures [29]. In general, the samples from experiments conducted under nitrogen (Fig. 6a) had higher absorbances than those treated under air (Fig. 6b), which suggests that high pressure promotes the decomposition of polymeric compounds, such as cellulose.

\subsection{Liquid composition}

The components of the liquid phase, which is bio-oil, were measured using HPLC methods. The main component of each sample was determined by quantifying each compound by comparing the measured results with standard samples. Generally, acetic acid, glycolic acid, and HMF were quantified using an absolute calibration curve method. Finally, hydrothermal experiments were conducted using standard samples of HMF and glycolic acid to elucidate the degradation pathway of the wood chips. In addition, glucose (14.96 min), fructose (15.53 $\mathrm{min})$, formic acid (18.63 min), levulinic acid (20.27) and ethanol (24.12 min) were also confirmed in liquid phase.

\subsubsection{Qualitative analysis by HPLC}

Wood chip hydrothermal liquefaction: It was determined that HMF was almost completely decomposed following hydrothermal treatments at temperatures over $180^{\circ} \mathrm{C}$. Regardless of the atmospheric conditions (air vs. nitrogen), the HMF peak observed in the HPLC chromatogram of the sample treated at $250^{\circ} \mathrm{C}$ decreased sharply relative to the sample treated at $180^{\circ} \mathrm{C}$. In addition, levulinic acid was detected in the $250^{\circ} \mathrm{C}$ sample. Therefore, we propose that $\mathrm{HMF}$ was decomposed in large quantities following a rehydration reaction at $250{ }^{\circ} \mathrm{C}$ to generate levulinic acid; such reactivity has been observed in previous studies [30]. To confirm this hypothesis, a standard HMF sample was treated by hydrothermal liquefaction at $250^{\circ} \mathrm{C}$. The HPLC 

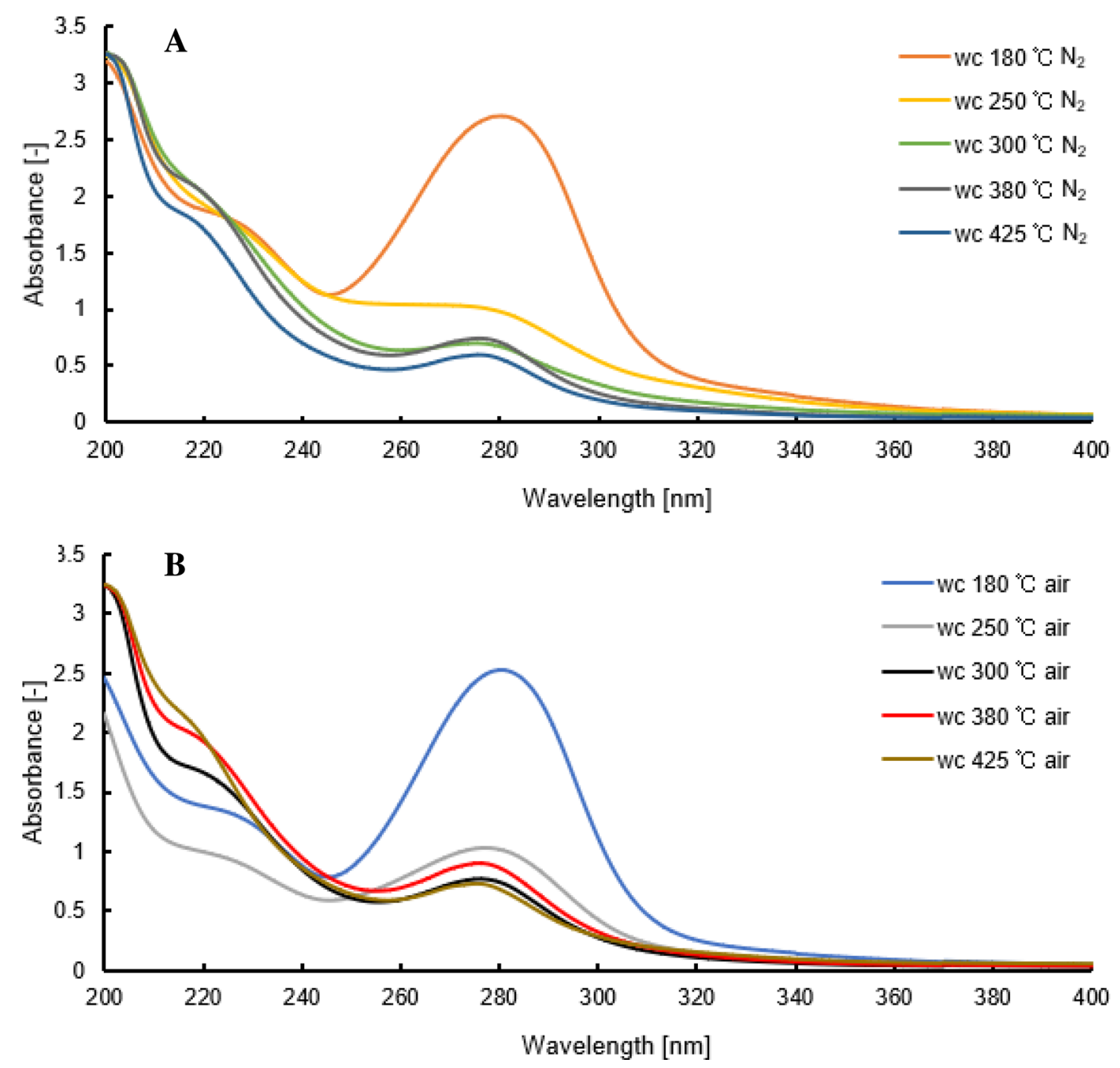

Fig. 6 UV absorbance profiles for each group of experiments (under $\mathrm{N}_{2}$ atmosphere (a) and air atmosphere (b))

results of this experiment showed that the main product of HMF decomposition after hydrothermal treatment at $250^{\circ} \mathrm{C}$ is indeed levulinic acid. Under a nitrogen atmosphere, a small amount of HMF was observed in the $250^{\circ} \mathrm{C}$ sample, in addition to glycolic acid and acetic acid, which were the main components. In contrast, it is believed that the glycolic acid is converted to acetic acid in the $380^{\circ} \mathrm{C}$ sample. The HPLC analysis of the sample obtained from supercritical processing (at $425^{\circ} \mathrm{C} ; \mathrm{N}_{2}$ ) is almost the same as that for the $380^{\circ} \mathrm{C}$ sample, both of which contain acetic acid as the main component. Based on these results, we conclude that acetic acid is the stable product of the hydrothermal reaction. In cases where acetic acid is formed, it is unlikely to decompose or react again. The glycolic acid concentrations in the $250^{\circ} \mathrm{C}$ and $300^{\circ} \mathrm{C}$ samples obtained under air atmosphere were significantly reduced compared with those in analogous experiments under a nitrogen atmosphere because glycolic acid reacts with oxygen in the air.

Hydrothermal liquefaction of standard samples: HTL experiments were conducted using standard samples of acetic acid, $\mathrm{HMF}$, and glycolic acid under $\mathrm{N}_{2}$ conditions to determine their decomposition products during hydrothermal treatments. Treating the standard HMF sample via HTL resulted in the formation of glycolic acid, formic acid, acetic acid, levulinic acid, and ethanol. Although levulinic acid and formic acid are produced in equal amounts following the rehydration of HMF, the concentration of levulinic acid is significantly higher than that of formic acid. The two elementary reactions involving formic acid dehydration and decarboxylation are shown in Eqs. 9 and 10, respectively [18, 21, 32]. These reactions lead to a much lower concentration of formic acid, relative to levulinic acid. After comparing the experimental results obtained 
at each treatment temperature, it was discovered that the degree of HMF decomposition increased significantly with treatment temperatures above $180^{\circ} \mathrm{C}$.

$\mathrm{HCOOH} \rightarrow \mathrm{CO}+\mathrm{H}_{2} \mathrm{O}$

$\mathrm{HCOOH} \rightarrow \mathrm{CO}_{2}+\mathrm{H}_{2}$

The qualitative results obtained after subjecting the standard glycolic acid sample to HTL treatment confirmed the production of acetic acid and a small amount of ethanol. Almost all glycolic acid was converted to acetic acid and gas after hydrothermal treatment at temperatures over $300^{\circ} \mathrm{C}$.

\subsubsection{Quantitative analysis}

The acetic acid, glycolic acid, and HMF produced after treatments were quantified using an absolute calibration curve method. All samples were analyzed twice using HPLC, and the average peak height for RI index was used for quantitative calculations.

Acetic acid: A calibration curve was constructed using standard acetic acid samples with concentrations of $0.3 \%$, $0.5 \%$, and $0.7 \%(\mathrm{v} / \mathrm{v})$. The results of the acetic acid quantification calculations performed for experimental samples are shown in Fig. 7. Since acetic acid is a stable product, it is difficult to decompose, so the yield of acetic acid yield is relatively unaffected by changes in temperature or atmospheric conditions. Based on the quantitative results, it was determined that the most suitable temperature for producing acetic acid was $300^{\circ} \mathrm{C}$; above this temperature, the decomposition rate of acetic acid exceeded its production rate, which caused reduced yields. The HPLC elution profile obtained from the treated wood chip sample with the highest acetic acid concentration $\left(300{ }^{\circ} \mathrm{C} ; \mathrm{N}_{2}\right)$ is shown in Fig. 8 a.

The highest concentration of acetic acid was measured for the liquid sample obtained after HTL treatment at $300^{\circ} \mathrm{C}$, and the ionic product of subcritical water at this temperature is also the largest [19]. This observation suggests that most of the reactions that produce acetic acid are ionic reactions, which are significantly affected by the ionic product of water. This relationship can also explain why the yield of acetic acid from experiments conducted under high-pressure nitrogen conditions were slightly higher than those in air conditions. The higher the pressure, the greater the ionic product [20].

Glycolic acid: Standard samples of glycolic acid with concentrations of $0.3 \%, 0.5 \%$, and $0.7 \%(\mathrm{v} / \mathrm{v})$ were used to produce a calibration curve. The glycolic acid quantifications of experimental samples are shown in Fig. 9. The concentration of glycolic acid decreased sharply when the temperature rose over $250{ }^{\circ} \mathrm{C}$, likely because glycolic acid can decompose at high temperatures. Additionally, the yield of this product following HTL treatment under a nitrogen atmosphere is higher than that under an air atmosphere because the glycolic acid reacts with oxygen in the air and converted to formic acid. The optimal conditions for producing glycolic acid are a temperature of $250^{\circ} \mathrm{C}$ and a nitrogen atmosphere, and the HPLC elution profile corresponding to these conditions is shown in Fig. 8b.

HMF: Standard samples of HMF at concentrations of $0.02 \%, 0.07 \%$, and $0.09 \%(\mathrm{v} / \mathrm{v})$ were used to construct a calibration curve. The HMF quantification results obtained for experimental samples are shown in Fig. 10. Based on these results, it was determined that the optimal conditions for producing HMF were a nitrogen atmosphere and a temperature of $180^{\circ} \mathrm{C}$; the $\mathrm{HPLC}$ elution profile obtained for this sample is shown in Fig. 8c. The concentration of HMF decreased significantly with treatment temperatures above $180^{\circ} \mathrm{C}$, regardless of the atmosphere. Interestingly, under a nitrogen atmosphere, HMF was not observed as a product in the sample treated at $300^{\circ} \mathrm{C}$, but when the treatment temperature exceeded $300^{\circ} \mathrm{C}, \mathrm{HPLC}$ analysis again confirmed the presence of HMF. This behavior is a result of the fact that the decomposition of HMF involves an exothermic reaction, and the reaction rate decreases as the temperature rises. In the early stage of the reaction, the concentration of glucose and fructose is high, so the production of HMF is faster than its decomposition. However, the concentrations of glucose and fructose decreased with increasing temperature, causing the production rate of HMF to fall below the decomposition rate and the yield of $\mathrm{HMF}$ to decline. After treatment at temperatures above $300^{\circ} \mathrm{C}$, the rate of HMF decomposition was suppressed by the high temperature, such that it became slower than the production rate; therefore, the HMF yield increased again thanks to the ionic product of the supercritical phase.

\subsection{Decomposition mechanism of wood chip pyrolysis}

The degradation pathway of the wood chips can be inferred based on the degradation products of glycolic acid and HMF (Fig. 11) [19-22, 33-35]. During the hydrothermal treatment reactions, cellulose is first decomposed to form glucose, which can be converted to fructose (which is more reactive) via isomerization. Fructose is decomposed to produce glycolic acid, and HMF can also be formed from the dehydration of fructose. HMF is primarily converted into levulinic acid and/or formic acid via rehydration reactions, whose reaction rates should increase significantly at temperatures above $180{ }^{\circ} \mathrm{C}$. Based on the hydrothermal experiments carried out using 

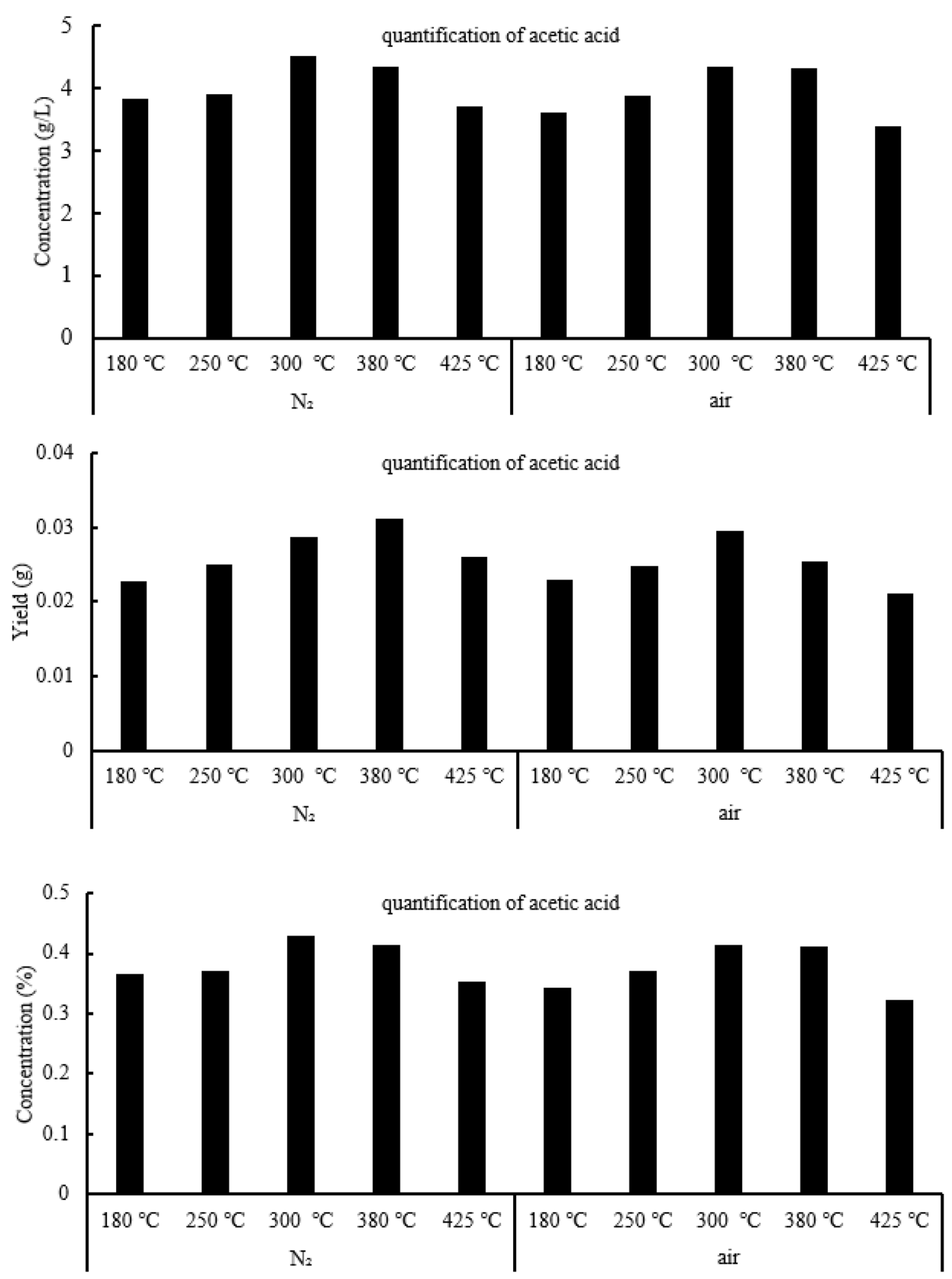

Fig.7 Quantification of acetic acid by its measured concentration (top; SD $<0.0501$ ) and yield (bottom; SD $<0.0003$ ) 

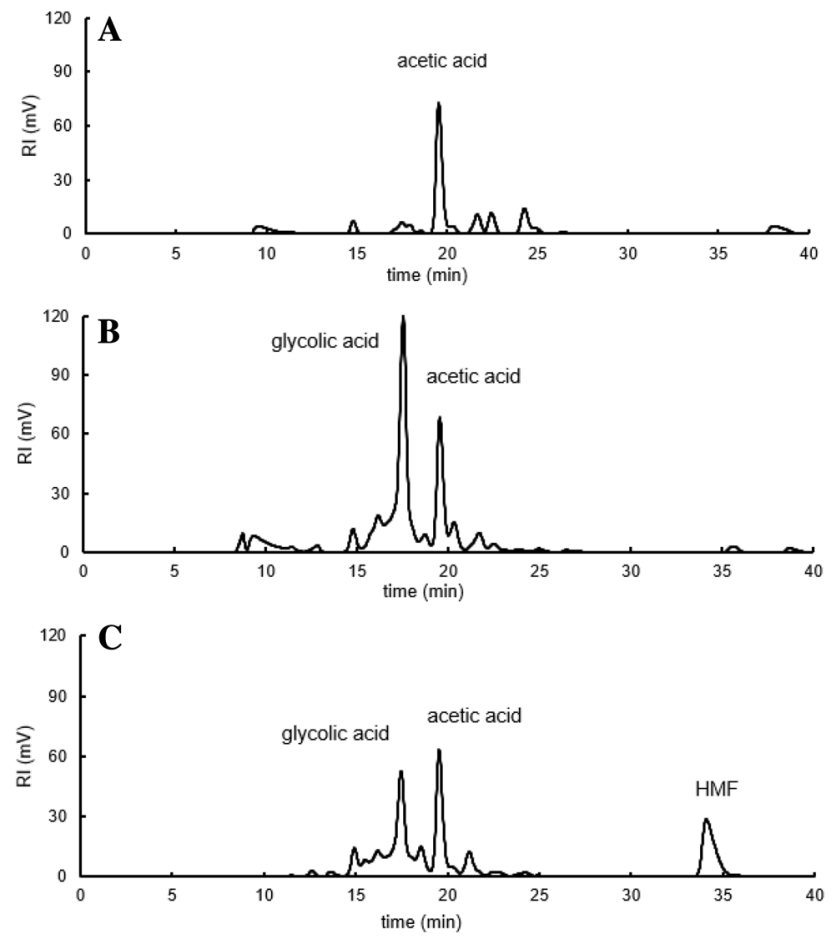

Fig. $8 \mathrm{HPLC}$ elution profiles from the liquid phase following wood chip pyrolysis. a $300^{\circ} \mathrm{C}, \mathrm{N}_{2} ;$ b $250^{\circ} \mathrm{C}, \mathrm{N}_{2} ; \mathbf{c} 180^{\circ} \mathrm{C}, \mathrm{N}_{2}$

standard HMF samples, it was discovered that acetic acid (in addition to levulinic acid and formic acid) can also be formed from HMF decomposition. Similarly, HTL treatments of glycolic acid standard samples demonstrated that glycolic acid could be converted into acetic acid when treated at temperatures over $250^{\circ} \mathrm{C}$. It is generally believed that hydrothermal reactions involving biomass ultimately produce acetic acid because of its high stability. However, when the reaction temperature exceeds $300^{\circ} \mathrm{C}$, the decomposition rate of acetic acid exceeds its rate of production, so under these conditions, the concentration of acetic acid in treated samples began to decrease.

\section{Conclusions}

Based on the results of hydrothermal liquefaction treatments of wood chips under various conditions, it was determined that a nitrogen atmosphere is generally favored for producing HMF, acetic acid, and glycolic acid. Quantitative HPLC analysis revealed that the nitrogen atmosphere promoted the generation of liquid phase products, partly because of the higher pressure used in these experiments, relative to those conducted under air. The air atmosphere is particularly not suitable for the production of glycolic acid. This may be because glycolic acid reacts with oxygen in the air to form formic acid, which shows that compared with air environment, nitrogen environment can inhibit oxidation reaction [36]. In general, the most suitable temperatures for producing acetic acid, glycolic acid, and $\mathrm{HMF}$, were $300^{\circ} \mathrm{C}, 250^{\circ} \mathrm{C}$, and $180^{\circ} \mathrm{C}$, respectively. Most papers suggest that $\mathrm{HMF}$ be produced at low temperatures, which is consistent with the conclusions of this study [26,37]. Interestingly, the yield of HMF first decreased, then increased as the temperature rose, and this behavior was attributed to the exothermic nature of the HMF decomposition reaction. Overall, HTL treatments under a high-pressure nitrogen atmosphere were tested using standard samples of acetic acid, glycolic acid, and HMF, and these conditions were shown to inhibit oxidation of the desired compounds at high temperatures.

Importantly, although conducting HTL treatments of biomass in air atmosphere can reduce the equipment costs and avoid the gas purging process, the results presented herein demonstrate that the concentration of each product following HTL in air is lower than those obtained after HTL in a nitrogen atmosphere. This is likely because of the potential reactions between the oxygen in air and the hydroxyl groups in the HTL products. Therefore, in terms of industrial production, we propose that nitrogen atmospheric conditions are preferred for controlling and optimizing the hydrothermal liquefaction of wood chip biomass resources. 

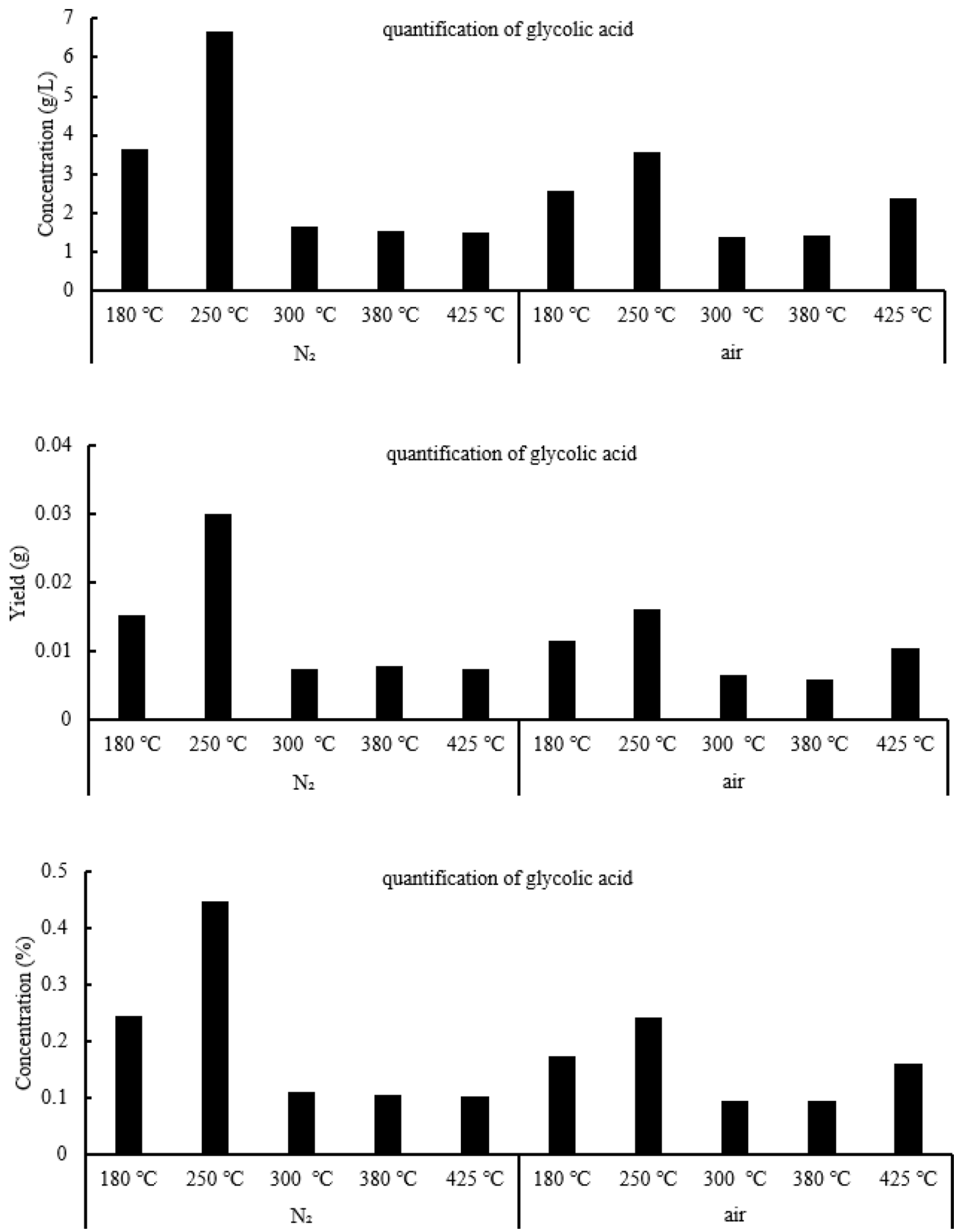

Fig.9 Quantification of glycolic acid by measured concentration (top; $S D<0.0261$ ) and by yield (bottom; SD $<0.0001$ ) 

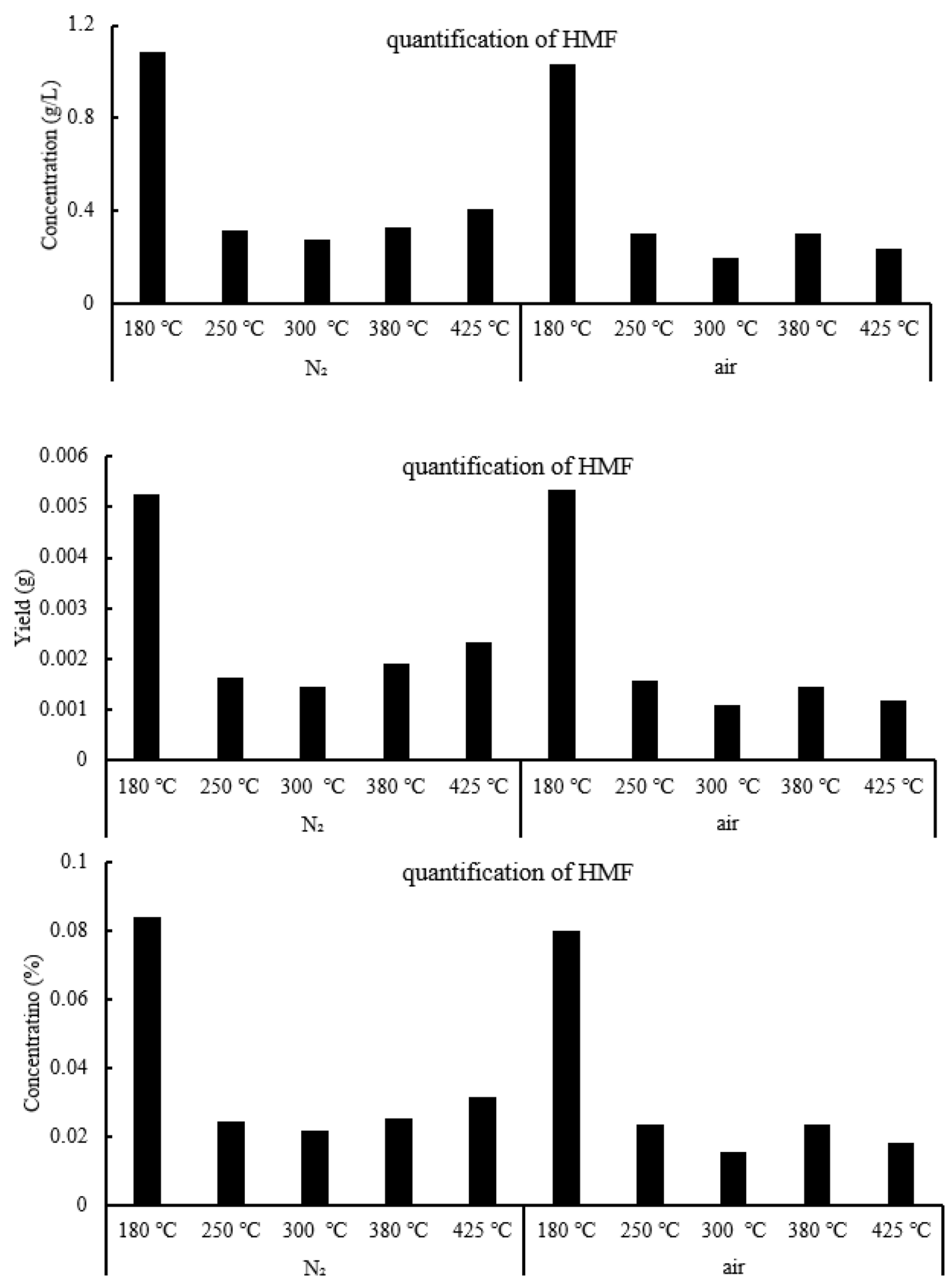

Fig. 10 Quantification of HMF by measured concentration (top; $\mathrm{SD}<0.0419$ ) and by yield (bottom; SD < 0.0002 ) 


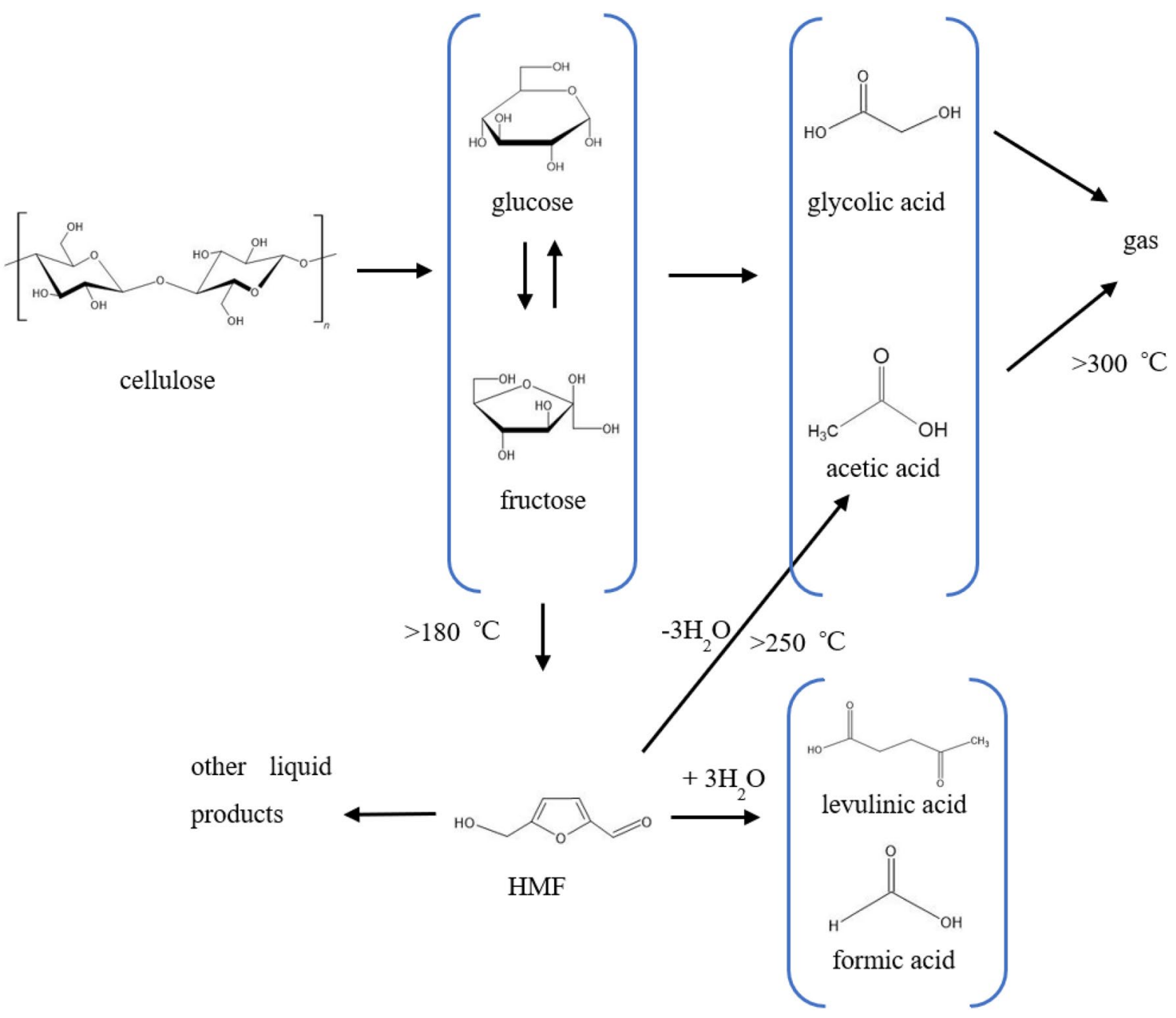

Fig. 11 Mechanism of wood chips hydrothermal liquefaction

Acknowledgements We thank Suzanne Adam, PhD, from Edanz Group (https://en-author-services.edanzgroup.com/ac) for editing a draft of this manuscript. The HPLC-RI analysis and TG-DTA analysis were performed by the instrumental analysis service of the Global Facility Center of Hokkaido University.

Author contributions N S: project design, methodology, and writing/ review. B Z: data analysis, writing the paper. K K: designed and conducted the experiment.

Data availability Relevant data are not shared.

\section{Declarations}

Conflicts of interest The authors declare that they have no conflicts of interest.

Open Access This article is licensed under a Creative Commons Attribution 4.0 International License, which permits use, sharing, adaptation, distribution and reproduction in any medium or format, as long as you give appropriate credit to the original author(s) and the source, provide a link to the Creative Commons licence, and indicate if changes were made. The images or other third party material in this article are included in the article's Creative Commons licence, unless indicated otherwise in a credit line to the material. If material is not included in the article's Creative Commons licence and your intended use is not permitted by statutory regulation or exceeds the permitted use, you will need to obtain permission directly from the copyright holder. To view a copy of this licence, visit http://creativecommons. org/licenses/by/4.0/.

\section{References}

1. Diaz JH (2007) The influence of global warming on natural disasters and their public health outcomes. Am J Disaster Med 2(1):33-42

2. Mohammed IS, Na R, Kushima K, Shimizu N (2020) Investigating the effect of processing parameters on the products of hydrothermal carbonization of corn stover. Sustainability 12(12):5100. https://doi.org/10.3390/su12125100

3. Ur-Rehman S, Mushtaq Z, Zahoor T, Jamil A, Murtaza MA (2015) Xylitol: a review on bioproduction, application, health benefits, and related safety issues. Crit Rev Food Sci Nutr 55(11):15141528. https://doi.org/10.1080/10408398.2012.702288

4. Ministry of agriculture, forestry and fisheries (2017) White paper on Forest and Forestry

\section{SN Applied Sciences}


5. Karagöz S, Bhaskar T, Muto A, Sakata Y, Oshiki T, Kishimoto T (2005) Low-temperature catalytic hydrothermal treatment of wood biomass: analysis of liquid products. Chem Eng J 108(12):127-137. https://doi.org/10.1016/j.cej.2005.01.007

6. Knezevic D, van Swaaij W, Kersten S (2010) Hydrothermal conversion of biomass. Il. Conversion of wood, pyrolysis oil, and glucose in hot compressed water. Ind Eng Chem Res 49(1):104-112. https://doi.org/10.1021/ie900964u

7. Yasaka M, Takitani M (2019) Sustainable forest management in an era of population decline. Forestry test news

8. Kambara S, Moritomi H (2009) Behavior of tar in pyrolysis and gasification of biomass. New Dev Biomass Technol 54:231-235

9. Braun M, Antonietti M (2017) A continuous flow process for the production of 2,5-dimethylfuran from fructose using (nonnoble metal based) heterogeneous catalysis. Green Chem 19(16):3813-3819. https://doi.org/10.1039/C7GC01055A

10. Wilson K, Lee AF (2014) Bio-based chemicals from biorefining: carbohydrate conversion and utilisation. Adv Biorefineries, Biomass Waste Supply Chain Exploit 19:624-658. https://doi.org/ 10.1533/9780857097385.2.624

11. Fritzsche CJF (1992) Nonpoint source pollution, calcium magnesium acetate deicer, an effective alternative for salt-sensitive areas. Water Environ Technol 45:44-51

12. Fangming J, Zhouyu Z (2005) Controlling hydrothermal reaction pathways to improve acetic acid production from carbohydrate biomass. Environ Sci Technol 39(6):1893-1902. https://doi.org/ 10.1021/es048867a

13. Wikberg $\mathrm{H}$, Ohra-aho $\mathrm{T}$, Honkanen $\mathrm{M}$, Kanerva $\mathrm{H}$, Harlin $\mathrm{A}$, Vippola M, Laine C (2016) Hydrothermal carbonization of pulp mill streams. Bioresour Technol 212:236-244. https://doi.org/10. 1016/j.biortech.2016.04.061

14. Madenoglu TG, Saglam M, Yüksel M (2013) Simultaneous effect of temperature and pressure on catalytic hydrothermal gasification of glucose. J Supercrit Fluids 73:151-160. https://doi.org/ 10.1016/j.supflu.2012.10.004

15. de Caprariis B, De Filippis P, Petrullo A, Scarsella M (2017) Hydrothermal liquefaction of biomass: influence of temperature and biomass composition on the bio-oil production. Fuel 208:618625. https://doi.org/10.1016/j.fuel.2017.07.054

16. Kruse Gawlik A (2003) Biomass conversion in water at 330-440 ${ }^{\circ} \mathrm{C}$ and $30-50 \mathrm{MPa}$, identification of key compounds for indicating different chemical reaction pathways. Ind Eng Chem Res 42(2):267-279. https://doi.org/10.1021/ie0202773

17. Möller M, Nilges $P$, Harnisch F, Schröder U (2011) Subcritical water as reaction environment: fundamentals of hydrothermal biomass transformation. Chem Sus Chem 4(5):566-579. https:// doi.org/10.1002/cssc.201000341

18. Yoshida K, Miyafuji H, Saka S (2010) Methane production from organic acids obtained by supercritical water treatment of Japanese beech. J Wood Sci 56(2):160-165. https://doi.org/10.1007/ s10086-009-1074-9

19. Asghari FS, Yoshida $\mathrm{H}$ (2006) Acid-catalyzed production of 5-hydroxymethyl furfural from d-fructose in subcritical water. Ind Eng Chem Res 45:2163-2173. https://doi.org/10.1021/ie051 088y

20. Yin SD, Tan ZC (2012) Hydrothermal liquefaction of cellulose to bio-oil under acidic, neutral and alkaline conditions. Appl Energy 92:234-239. https://doi.org/10.1016/j.apenergy.2011. 10.041

21. Dolan R, Yin SD, Tan ZC (2010) Effects of headspace fraction and aqueous alkalinity on subcritical hydrothermal gasification of cellulose. Int J Hydrog Energy 35:6600-6610. https://doi.org/ 10.1016/j.ijhydene.2010.04.029
22. Möller M, Nilges $P$, Harnisch F, Schröder U (2011) Subcritical water as reaction environment: fundamentals of hydrothermal biomass transformation. Chem Sus Chem 4:566-579. https:// doi.org/10.1002/cssc.201000341

23. Iwamura H, Sato T, Okada M, Sue K, Hiaki T (2014) Organic reactions in sub-and supercritical water in the absence of any added catalyst. J Res Inst Sci Tech Nihon Univ 132:1-9. https://doi.org/ 10.11346/cstj.2014.132_1

24. Ishak H, Yoshida H, Muda NA, Ismail MHS, Izhar S (2019) Rapid processing of abandoned oil palm trunks into sugars and organic acids by sub-critical water. Processes 7:593. https://doi. org/10.3390/pr7090593

25. Yoshida H, Izhar S, Nishio E, Utsumi Y, Kakimori N, Feridoun SA (2018) Application of sub-critical water for recovery of tin and glass substrates from LCD panel E-waste. Detritus. https://doi. org/10.31025/2611-4135/2018.13738

26. Asghari FS, Yoshida $\mathrm{H}$ (2010) Conversion of Japanese red pine wood (Pinus densiflora) into valuable chemicals under subcritical water conditions. Carbohydr Res 345:124-131. https://doi. org/10.1016/j.carres.2009.10.006

27. Promdej C, Matsumura Y (2011) Temperature effect on hydrothermal decomposition of glucose in sub-and supercritical water. Ind Eng Chem Res 50(14):8492-8497. https://doi.org/10. 1021/ie200298c

28. Hudz N, Leontiev D, Wieczorek PP (2019) Spectral characteristics of 5-hydroxymethylfurfural as a related substance in medicinal products containing glucose. Pharm 66:121. https://doi.org/10. 3897/pharmacia.66.e35969

29. General rules for absorptiometric analysis JIS K0115 (2004)

30. Falco C, Baccile N, Titirici MM (2011) Morphological and structural differences between glucose, cellulose and lignocellulosic biomass derived hydrothermal carbons. Green Chem 13:3273. https://doi.org/10.1039/C1GC15742F

31. Yu J, Savage PE (1998) Decomposition of formic acid under hydrothermal conditions. Ind Eng Chem Res 37(1):2-10. https:// doi.org/10.1021/ie970182e

32. Watanabe $M$, Sato $T$, Inomata $H$, Smith RL Jr, Arai $K$ Jr, Kruse $A$, Dinjus E (2004) Chemical reactions of $C 1$ compounds in nearcritical and supercritical Water. Chem Rev 104(12):5803-5822. https://doi.org/10.1021/cr020415y

33. Chuntanapum A, Yong TLK, Miyake S, Matsumura Y (2008) Behavior of 5-HMF in subcritical and supercritical water. Ind Eng Chem Res 47:2956-2962. https://doi.org/10.1021/ie0715658

34. Assary RS, Redfern PC, Hammond JR, Greeley J, Curtiss LA (2010) Computational studies of the thermochemistry for conversion of glucose to levulinic acid. J Phys Chem B 114:9002-9009. https://doi.org/10.1021/jp101418f

35. Jin F, Zhou Z, Kishita A, Enomoto H (2006) Hydrothermal conversion of biomass into acetic acid. J Mater Sci 41:1495-1500. https://doi.org/10.1007/s10853-006-7493-8

36. Tolbert NE, Clagett CO, Burris RH (1949) Products of the oxidation of glycolic acid and L-lactic acid by enzymes from tobacco leaves. J Biol Chem 181(2):905-914. https://doi.org/10.1016/ S0021-9258(18)56614-X

37. Takeuchi Y, Jin F, Tohji K, Enomoto H (2018) Acid catalytic hydrothermal conversion of carbohydrate biomass into useful substances. J Mater Sci 43(7):2472-2475. https://doi.org/10.1007/ s10853-007-2021-z

Publisher's Note Springer Nature remains neutral with regard to jurisdictional claims in published maps and institutional affiliations. 